\title{
Optimising zero-order suppression in ion-exchanged phase gratings
}

\author{
Carlos Montero-Orille ${ }^{1, *}$, Héctor González-Núñez ${ }^{1}$, Xesús Prieto-Blanco ${ }^{1}$, Vicente Moreno ${ }^{1}$, Dolores Mouriz ${ }^{1}$, María C. \\ Nistal $^{1}$, and Jesús Liñares ${ }^{1}$ \\ ${ }^{1}$ Quantum Materials and Photonics Group, Department of Applied Physics, Faculty of Physics / Faculty of Optics and Optometry, \\ University of Santiago de Compostela, Campus Vida s/n, E-15782, Santiago de Compostela, Galicia, Spain
}

\begin{abstract}
Ion-exchange in glass is a well-known technique to fabricate phase optical elements. For elements with reduced dimensions, the side diffusion, intrinsic to ion-exchange processes, can affect the performance of these elements if it is not taken into account. Here we present a procedure to optimise the zero-order suppression of ion-exchanged phase gratings.
\end{abstract}

\section{Introduction}

Ion-exchanged phase gratings are very versatile optical elements [1]. They can be used in spectrometry, wavelength division multiplexing, power division or optical pulse compression. On the other hand, ion-exchange is a well established technique to modify the refractive index of a substrate. This modification can be made in different areas by using appropriate masks. Ion-exchange has several advantages, namely, simplicity, low cost and easy control of the fabrication parameters. Moreover, the flat surface of the elements fabricated by ion-exchange permits straightforward cleaning and antireflection coating. The fabrication of high performance elements with reduced dimensions by ion-exchange is a delicate task. In particular, it is essential to take into account the side diffusion inherent to any thermal ion-exchange process which produces a transition region [2]. If this is not done, the phase profile of the grating as well as its performance will be affected.

Here we present the design and fabrication by ionexchange of a phase diffraction grating with its zero-order suppressed. Removal of the zero-order of diffraction is desirable in most applications, including digital holography, spatial light modulation and grating fabrication [3, 4]. As it is known, a binary phase grating does not have zeroorder if its phase profile is antisymmetric and its phase step is $\pi$. However, this is not the case for an ion-exchanged grating due to the mentioned side diffusion (see Fig. 1). Therefore, in this work, we show how to compensate the phase introduced in the transition region by a proper design of the ion-exhange process and the mask (lithographical process) used to produce the diffraction grating.

\section{Materials and Methods}

Soda-lime glasses of $1 \mathrm{~mm}$ thickness and refractive index $n_{s}=1.5103$, for $\lambda=632.8 \mathrm{~nm}$, have been used as sub-

*e-mail: carlos.montero@usc.es strates. The grating fabrication was made in these substrates by thermal ion-exchange and using aluminium layers as a mask for the ions. A binary grating pattern was translated from a sheet of heavy paper to the substrate by a two-step photolithographic process. The binary pattern was designed taking into account the side diffusion inherent to the ion-exchange process. The first photolithographic reduction was made by a lens Schneider XENON Sapphire 4.5/95, using a magnification of $M=-1 / 14.1$. In the second step a Zeiss S-Planar 1.1/68 lens performing a reduction 1:5 was used. The final result was an aluminium binary grating on the substrate. As for the ion exchanges, we used a salt mixture $5 \%$ mole $\mathrm{AgNO}_{3} / \mathrm{NaNO}_{3}$ at $340{ }^{\circ} \mathrm{C}$. The ion-exchange modification produced by this salt in these glasses was analysed by using the in-
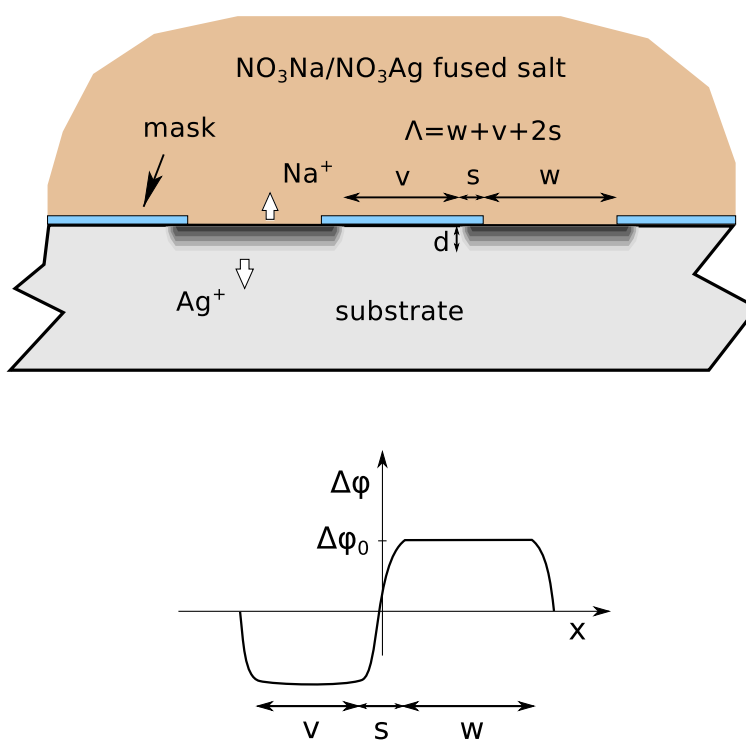

Figure 1. Scheme of an ion-exchanged grating and its phase profile. The side diffusion gives rise to a gradient in the phase profile. 

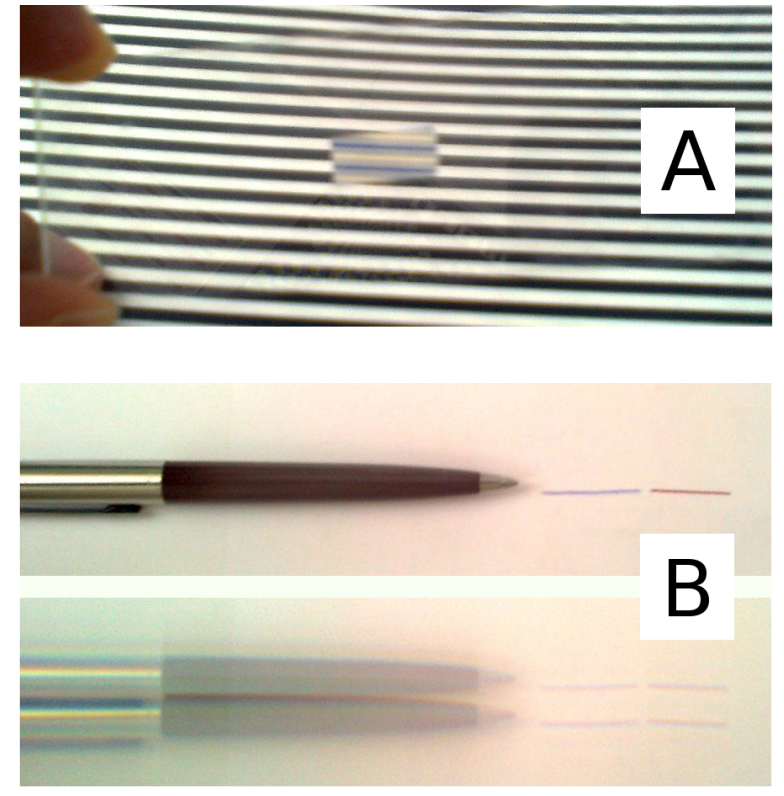

Figure 2. Image through one of the gratings fabricated of: A) a black and white object and B) a long object.

verse Wentzel-Kramers-Brillouin (IWKB) method. As it is known, this method recovers the gradient index profile following an ion-exchange process, which can be represented by a function $n=n_{s}+\Delta n(x / d)$ for a 1D ionexchange, with $d$ an effective depth [5]. After determination and integration of this profile, the phase step $\left(2 \Delta \varphi_{0}\right)$ generated by the ion-exchange can be calculated.

With regard to the design of the grating, we recall that our goal was to minimise its zero-order. As we said, the phase profile must be antisymmetric for this purpose. This means $w=v$ (see Fig. 1) and hence the period of the grating must be $\Lambda=2(w+s)$. On the other hand, due to the isotropic nature of the ion exchange, the size of the side diffusion $(s)$ is similar to the size of the in-depth diffusion $(s \approx d)$. Moreover, both effective depths are proportional to the maximum phase step $\left(2 \Delta \varphi_{0}\right)$ and this phase step must be set properly. Indeed, for a moderate side diffussion, we must look for a maximum phase step slightly bigger than $\pi$ to compensate for the minor phase step that the side region gives. Therefore, the design of both the aluminium mask and the ion-exchange process are linked and must be done together. After fabrication of the aluminium mask, we fabricated several gratings by changing the ionexchange time in order to find the optimal one: the grating with the minimum zero-order. The performance of these gratings was assessed by measuring the efficiency of their zero-order through two methods: 1) directly, by using a $\mathrm{He}-\mathrm{Ne}$ laser and a power meter, and 2) by measuring their spectral transmittance with a spectrometer.

\section{Experimental results}

We fabricated two gratings designed to suppress the zeroorder at $\lambda=632.8 \mathrm{~nm}$. We chose their periods to be $\Lambda=60$ $\mu \mathrm{m}$ and $\Lambda=30 \mu \mathrm{m}$. Hence their dimensions were $w=v=25$ $\mu \mathrm{m}$ and $w=v=10 \mu \mathrm{m}$, respectively, because we estimated that an in-depth diffusion $d \approx 5 \mu \mathrm{m}$ was needed. In order to look for the properly phase step, a calibration of the ionexchange process was made based on the diffusion time [5]. We found that an ion-exchange time of $t=12.6 \mathrm{~min}$ gives rise to $2 \Delta \varphi_{0}=\pi$ in the glasses used. Because a bigger than $\pi$ maximum phase step should be necessary to minimise the zero-order, we made several gratings with longer times and measured their performance by the two methods commented in the previous section. We found that ion-exchange times of $t=15.5 \mathrm{~min}$, for the grating with period $\Lambda=60 \mu \mathrm{m}$, and $t=28.2 \mathrm{~min}$, for the grating with period $\Lambda=30 \mu \mathrm{m}$, gave the best results: gratings with a zero-order efficiency $T_{0}<0.5 \%$. The phase steps of both gratings were $2 \Delta \varphi_{0}=1.11 \pi$ and $2 \Delta \varphi_{0}=1.52 \pi$, respectively, supporting these results our initial hypothesis.

We show, in Fig. 2, the image of two objects through the grating of $\Lambda=30 \mu \mathrm{m}$. In Fig. $2 A$ the grating can be seen in the central rectangular region of the microscope slide used as a substrate, where the zero-order suppression is observed. The white region of the object that is behind becomes blue at the zero-order, because we did the design for red light. On the other hand, Fig. 2B shows a pen without (up) and with (down) the grating in front of the camera lens. The double image proofs that the \pm 1 orders are the most relevant ones.

\section{Conclusion}

We have presented the fabrication by thermal ionexchange of phase gratings with their zero-order suppressed. The design of these gratings has been made by taking into account the side diffusion inherent to any thermal ion-exchange process. In particular, a specific design of the mask needed for the fabrication must be done. The removal of the zero-order that we achieved is excellent: its efficiency falls below $0.5 \%$.

Funding. This research was funded by the 'Ministerio de Economía y Competitividad', Central Government of Spain, grant number AYA2016-78773-C2-2-P, the 'Fondo Europeo de Desenvolvemento Rexional' (FEDER), and by 'Consellería de Educación, Universidade e FP, Xunta de Galicia', under Grant GRC No. ED431C 2018/11.

\section{References}

[1] R.P. Salmio, J. Saarinen, Applied Physics Letters 66, 917 (1995).

[2] J. Liñares, E. López-Lago, C. Montero and X. Prieto, Pure Appl. Opt.A, 5, 919 (1996)

[3] W.D.G.D. Improso, G.A. Tapang, C.A. Saloma, Suppression of Zeroth-Order Diffraction in Phase-Only Spatial Light Modulator, Vol. 222 of Springer Series in Optical Sciences (Springer, Cham, 2019).

[4] S.J. Mihailov, C.W. Smelser, P. Lu, R.B. Walker, D. Grobnic, H. Ding, G. Henderson, J. Unruh, Opt. Lett. 28, 995 (2003).

[5] X. Prieto-Blanco, C. Montero-Orille, V. Moreno, E.F. Mateo, J. Liñares, Applied optics 54, 3308 (2015). 